\title{
Quantum Charge Transfer Study of Triply Charged Ions in the Adiabatic Representation: the $(B H e)^{3+}$ System
}

\author{
A. López-Castillo*, J.C. Belchior, and J.P. Braga \\ Departamento de Química, ICEx, Universidade Federal de Minas Gerais, \\ 31270-901 Belo Horizonte - MG, Brazil
}

Received: August 29, 1997

\begin{abstract}
O estudo quântico do processo $\mathrm{B}^{3+}+\mathrm{He} \rightarrow \mathrm{B}^{2+}+\mathrm{He}^{+}$foi investigado para energias de colisão variando de 1-102 eV usando um potencial de interação ab-initio. Um método novo foi usado para resolver a equação de Schrödinger na base adiabática, onde os acoplamentos rotacional e radial foram considerados. Além disso, uma discussão sobre os acoplamentos de diferentes simetrias é apresentada. Usando o modelo de Landau-Zenner, mostramos que o modelo de dois estados não deve ser usado para este sistema. Este estudo poderia indicar que tal modelo deve ser usado cuidadosamente para outros sistemas onde o processo de transferência de carga seja considerado. Finalmente, as seções de choque total quântica foram comparadas com trabalhos publicados anteriormente por Gargaud e co-autores e uma concordância razoável foi observada.

Full quantum charge transfer study of the process $\mathrm{B}^{3+}+\mathrm{He} \rightarrow \mathrm{B}^{2+}+\mathrm{He}^{+}$has been investigated in the collision energy range $1-102 \mathrm{eV}$ using an ab-initio interaction potential. A new method to solve the Schrödinger equation in an adiabatic basis was used, where the radial and rotational coupling were taken into account, and the importance of the coupling between states of different symmetry was discussed. Moreover, by using the well known Landau-Zener model, it was concluded that the two state model cannot be applied for the present system, and this might indicate that such a model should be applied carefully for other systems when a charge transfer process is considered. Finally, the quantum total cross sections were compared with the previous published work of Gargaud and co-workers and a fair agreement was achieved.
\end{abstract}

Keywords: charge transfer, quantum scattering, cross sections

\section{Introduction}

Charge transfer in atomic and molecular collisions has been intensively studied in the past few decades ${ }^{1-5}$. The importance of this process arises in different branches of science such as astrophysics, chemistry, laser research, material science and many others 5 .

Theoretical or experimental works in this field have grown up rapidly due to new techniques that have become ${ }^{6}$ available, especially in the case of high resolution molecular beam experiments ${ }^{7}$.

From the theoretical point of view the mechanisms to elucidate charge transfer processes need accurate potential energy functions and therefore exact quantum calculations have to be carried out. However, most charge transfer processes studied have been mainly concerned with only single charge transfer systems ${ }^{5}$. In this case only covalent dissociating states are involved and hence the quantum scattering calculations are simpler. On the other hand, for systems with multiple charge transfer such calculations are more difficult to perform due to covalent and ionic states being involved. The first quantum theoretical work where double charges were considered was that of Braga et al. ${ }^{8}$ In the latter work, the $A r^{2+} / \mathrm{He}$ system was analysed by quantum mechanics and an intermolecular potential model was proposed based on ab-initio calculations. The quantum scattering calculations were carried out in the diabatic basis and no rotational to radial couplings were included. Nevertheless satisfactory agreement was achieved compared with the experimental measurements of Friedrich and collaborators 9 . 
The recent research review of Herman ${ }^{10}$ has updated the existing literature on charge transfer in atomic and molecular collisions in the experimental and theoretical pictures. As shown by Herman, the experimental apparatus have shown better resolution and consequently lower relative errors can be obtained for the experimental data.

For the charge transfer process with doubly charged molecules, Herman and co-workers ${ }^{10-11}$ have shown that theory and experiment are well developed for resolving excited electronic states. For example, Herman and collaborators ${ }^{11}$ have studied the $\mathrm{CO}^{2+}+\mathrm{Ne}$ system by using the potential energy function calculated by Larsson et al. ${ }^{12}$ for this single charge transfer process. This latter work showed a quantitative agreement with recent experiments of cross molecular beams perform by Hamdan and Brenton ${ }^{13}$, and has confirmed that the intermolecular potential of Larsson et al. ${ }^{12}$, for $\mathrm{CO}^{2+} / \mathrm{Ne}$ collision is able to describe the energy separation of the minima and the equilibrium distance for the $1^{3} \Pi$ and $1^{3} \Sigma$ states. However, due to the resolution of the energy, about $0.3 \mathrm{eV}$, the vibrational states were not fully resolved although the important features of the population for the energy spectra were analysed. For polyatomic molecules, such as $\mathrm{NH}_{3}$ and $\mathrm{H}_{2} \mathrm{~S}$ colliding with $\mathrm{He}^{2+}$, the theoretical and experimental work of Fárník and co-work$\mathrm{ers}^{14}$ are the most recent advance in multiple charge transfer processes. By using the crossed molecular beam technique with resolution of about $120 \mathrm{meV}$, they were able to study, in detail, the contribution of the energy transfer between all quantum state. The relaxation into each rotational, vibrational and electronic states has provided important results such as the Franck-Condon factors.

According to Herman ${ }^{10}$, simple models have been applied to dication-molecule and cation-molecule chemical reactions and charge transfer processes. In addition, charge transfer processes with multiple charge are one of the research areas that have recently turned out to be of considerable interest.

Very recently Boyd and collaborators ${ }^{15}$ have proposed an inversion of the diabatic coupling potentials for multiple charge transfer on the $\mathrm{C}^{4+} / \mathrm{He}$ system. Experimental inelastic cross sections were used as the input data. By using a general inversion algorithm based on functional sensitive analysis, the diabatic coupling potential for such a system was derived. This inversion was accurate especially in the

$$
\begin{aligned}
& \quad \frac{\mathrm{d}^{2}}{\mathrm{dR}^{2}} \mathbf{u}(R)+\mathbf{Q}(R) \mathbf{u}(R)=0 \\
& B^{2+}\left(1 s^{2} 2 s\right)+H e^{+}(1 s), 1^{1} \Sigma^{+} \\
& B^{3+}\left(1 s^{2}\right)+\mathrm{He}\left(1 s^{2}\right), 3^{1} \Sigma^{+} \rightarrow \quad \\
& B^{2+}\left(1 s^{2} 2 p\right)+H e^{+}(1 s), 2^{1} \Sigma^{+} \\
& B^{2+}\left(1 s^{2} 2 p\right)+H e^{+}(1 s), 1^{1} \Pi \\
& B^{2+}\left(1 s^{2} 3 s\right)+H e^{+}(1 s), 4^{1} \Sigma^{+}
\end{aligned}
$$
perform. set $^{17}$. experimental results of the Iwai et al. ${ }^{20}$

\section{Adiabatic quantum scattering theory} in the diabatic basis,

The present work will analyse the charge transfer of the $\mathrm{B}^{3+} / \mathrm{He}$ system, which is isoelectronic to $\mathrm{C}^{4+} / \mathrm{He}$, and where ionic and covalent states are also involved. López-Castillo and Ornellas ${ }^{16,17}$ have recently produced a potential energy surface where all the important couplings (rotational and radial) for the dynamics were well defined. The reactions presented in Eq. 1 were calculated with an accurate basis

Although the above authors have produced quality potentials and couplings, their ab-initio calculations have been tested using the classical path method ${ }^{16}$ for colinear trajectories and for very high collision energy ( $1 \mathrm{keV}$ up to $50 \mathrm{keV})$. Nevertheless, their calculations are in fair agreement with experimental cross sections, but some experimental data ${ }^{18,19}$ are overestimated. The agreement is almost qualitative, but it is quite consistent with the subsequent

This paper aims to calculate the state-to-state total cross sections for the above process, in the collision energy ranging from $1 \mathrm{eV}$ to $102 \mathrm{eV}$ and using the renormalized Fox-Goodwin method as given by Braga and Belchior ${ }^{21}$. The latter method provides a powerfull tool to propagate the radial wavefunction with the intermolecular potential in an adiabatic basis. This combination of low collision energy and quantum analysis will provide a better understanding of the quality of the potential energy surfaces given by López-Castillo and Ornellas ${ }^{16}$. In addition, the well known two state Landau-Zener (LZ) model will be applied to analyse the validity of approaching a problem of several states by a two state model. Such a method is well detailed elsewhere ${ }^{8}$ and will not be repeated here. Since there is no experimental data in this collision energy range, a comparison with previous theoretical work will be done $^{22}$. Actually, the low collision energy used in the present calculations could be achieved by experimentalists for carrying out measurements in this energy range.

The matrix representation of the Schrödinger equation 
where

$$
\mathbf{Q}(R)=\mathbf{k}^{2}-\frac{l(l+1)}{R^{2}} \mathbf{I}-\frac{2 \mu}{\hbar^{2}} \mathbf{V}(R)
$$

$\mathbf{k}^{2}$ being the channel wave number, $l$ the angular momentum, $\mu$ the system reduced mass and $\mathbf{V}(R)$ the intermolecular potential, is not always convenient to use in the study of charge transfer process. The non-commutability of the rotational to radial operators prevents the above representation from always be useful. In a standard procedure rotational to radial couplings are neglected and the diabatic representation is used for studying charge transfer dynamics.

Concerned with the above problem Braga and Belchior $^{21}$ have recently developed an algorithm to integrate the Schrödinger equation in the adiabatic representation, which is defined in such a way that, in this new representation, the diabatic potential becomes diagonal. Certainly this is performed with the expense of making the kinetic operator non-diagonal in this adiabatic representation. Nevertheless the two representations should coincide for the large scattering coordinate where the transformation between these two representations, denoted by $\mathbf{U}(R)$, becomes $R$ independent and goes to a constant equals to the identity transformation.

In this new representation the Schrödinger equation is then written as,

$$
\begin{gathered}
\frac{\mathrm{d}^{2}}{\mathrm{dR}^{2}} \mathbf{X}(R)+2\left(\mathbf{U}^{-1}(R) \frac{\mathrm{d}}{\mathrm{dR}} \mathbf{U}(R)\right) \frac{\mathrm{d}}{\mathrm{dR}} \mathbf{X}(R)+ \\
\left(\mathbf{U}^{-1}(R) \frac{\mathrm{d}^{2}}{\mathrm{dR}^{2}} \mathbf{U}(R)\right) \frac{\mathrm{d}}{\mathrm{dR}} \mathbf{X}(R)+ \\
\left(\mathbf{k}^{2}-\frac{l(l+l)}{R^{2}} \mathbf{I}-\frac{2 \mu}{\hbar^{2}} \mathbf{D}(R)\right) \mathbf{X}(R)=0
\end{gathered}
$$

The algorithm to solve the above equation ${ }^{21}$, which combines efficiency and simplicity, is based on a three point recurrence relation established by Fox and Goodwin in $1949^{23}$. Defining the quantities,

$$
\begin{gathered}
\mathbf{Q}_{\mathrm{A}}(R)=\mathbf{k}^{2}-\frac{l(l+l)}{R^{2}} \mathbf{I}-\frac{2 \mu}{\hbar^{2}} \mathbf{D}(R)+ \\
\mathbf{U}^{-1}(R) \frac{\mathrm{d}^{2}}{\mathrm{dR}^{2}} \mathbf{U}(R)
\end{gathered}
$$

and,

$$
\mathbf{P}(R)=2\left(\mathbf{U}^{-1}(R) \frac{\mathrm{d}}{\mathrm{dR}} \mathbf{U}(R)\right)
$$

the adiabatic Schrödinger equation is then transformed ${ }^{21}$ into the recurrence relation,

$$
\begin{aligned}
& \mathbf{R}(R+h)=\left(\mathbf{I}+\frac{h}{2} \mathbf{P}(R)\right)^{-1}\left(2 \mathbf{I}-h^{2} \mathbf{Q}_{\mathrm{A}}(R)\right)- \\
&\left(\mathbf{I}+\frac{h}{2} \mathbf{P}(R)\right)^{-1}\left(\mathbf{I}-\frac{h}{2} \mathbf{P}(R)\right)^{-1} \mathbf{R}^{-1}(\mathrm{R}-h)
\end{aligned}
$$

where $\mathbf{R}(R)=\mathbf{X}(R+h) \mathbf{X}^{-1}(R)$. This renormalization gives the necessary stability for the closed channels as discussed in detail by Braga and Belchior ${ }^{21}$. The matrices $\mathbf{U}(R)$ and $\mathbf{D}(R)$ are provided by the electronic calculations. A bicubic spline interpolator was implemented with the quantum scaterring code and hence no analytical fitting of these matrices were necessary. For details about the electronic calculation see references ${ }^{16,17}$.

In the asymptotic region the Schrödinger equation, either in the adiabatic or the diabatic representation, is decoupled. Nevertheless the ionic and covalent channels have to be matched with different boundary conditions due to the tail of the coulomb potential that decreases slower than the centrifugal term. These equations are then given, for the large scattering coordinate, as

$$
\left(\frac{\mathrm{d}^{2}}{\mathrm{~d} \rho_{\mathrm{i}}^{2}}+1-\frac{l(l+l)}{\rho_{\mathrm{i}}^{2}}-2 \frac{\eta}{\rho_{\mathrm{i}}}\right) X_{i}\left(\eta, \rho_{\mathrm{i}}\right)=0
$$

where $\rho_{i}=k_{i} R$ and

$$
\eta=\frac{\mu}{\hbar^{2}} \frac{Z_{1} Z_{2} \mathrm{e}^{2}}{k_{i}}
$$

For the covalent channel this quantity has to be taken equal to zero.

The Riccati-Bessel functions were generated by using forward and backward recurrence relations ${ }^{24}$ whereas the Coulomb functions were generated using Steed's algorithm in complex form ${ }^{25}$. Denoting the diagonal matrices that contain the Riccati-Bessel and Coulomb functions of the first kind and second kind respectively by $\mathbf{J}(R)$ and $\mathbf{N}(R)$ the $\mathbf{K}$ matrix can then be obtained by,

$$
\begin{aligned}
& \mathbf{K}=(\mathbf{R}(R) \mathbf{N}(R)-\mathbf{N}(R+\mathrm{h}))^{-1}(\mathbf{R}(R) \mathbf{J}(R)- \\
& \mathbf{J}(R+\mathrm{h}))
\end{aligned}
$$

Scattering matrices and cross sections are then obtained in the usual way $^{8}$, i.e, an average over all partial waves are given by

$$
\sigma_{i \rightarrow j}=\frac{\pi}{k_{\mathrm{i}}^{2}} \sum_{l=0}^{\infty}(2 l+1)\left|S_{i j}^{l}\right|^{2}
$$

where $S_{i j}{ }^{l}$ is the scattering matrix for each partial wave and for the transition $i$ to $j$.

\section{The interaction potential}

To solve the Hartree-Fock-Roothaan equation, initially one has to select a set of atomic basis functions for the molecular expansion. These functions are normally ex- 
pressed as a linear combination of cartesian Gaussians, which are easier to work in numerical calculations.

The basis sets selected for the $[\mathrm{BHe}]^{3+}$ system are of dupla-zeta type and are described in Refs. 26 and 27. They also contain $p$-type and $d$-type polarization functions for the $H e$ and $B$ atoms respectively. They are represented by $(11 s, 6 p) /[5 s, 4 p]$ for boron, with three $d$-type polarization function with coefficients $1.11,0.402$ and $0.145^{26}$ and $(6 s, 1 p) /[4 s, 1 p]$ for helium ${ }^{27}$. The electronic structure calculation was performed using the MELD program ${ }^{28}$ and the potential energy curves were then calculated in the adiabatic approximation for the quasi-molecule $[\mathrm{BHe}]^{3+}$ in the configuration interaction (CI) level. Single and double excitations relative to the Hartree-Fock configuration $\left(B^{3+}\left(1 s^{2}\right)+H e^{0}\left(1 s^{2}\right)\right)$ were therefore generated. For more details see Ref. 16.

The radial and rotational couplings can be formally written as

$$
\begin{aligned}
& <\Psi_{m}\left|\frac{\partial}{\partial R}\right| \Psi_{n}>=\sum_{i} C_{i}^{m} \frac{\partial C_{i}^{n}}{\partial R}+ \\
& \sum_{\alpha \beta} P_{\alpha \beta}^{m n}\left(\sum_{p \neq q} a_{p}^{\alpha}\left[a_{q}^{\beta}<\varphi_{p}\left|\frac{\partial}{\partial R}\right| \varphi_{q}>\right]+<\varphi_{p} \mid \varphi_{q}>\frac{\partial \mathrm{a}_{q}^{\beta}}{\partial R}\right]+ \\
& \sum_{p} a_{p}^{\alpha} \frac{\partial \mathrm{a}_{p}^{\beta}}{\partial R} \\
& \text { and } \\
& <\Psi_{m}\left|i L_{y}\right| \Psi_{n}>=\sum_{\alpha \beta} P_{\alpha \beta}^{m n} \sum_{p q} a_{p}^{\alpha} \\
& a_{q}^{\beta}<\varphi_{p}\left|i L_{y}\right| \varphi_{q}>
\end{aligned}
$$

where $\Psi_{n}$ is a CI wave function, $\mathrm{C}_{i}{ }^{n}$ are the CI coefficients, $\mathrm{P}_{\alpha \beta}{ }^{m n}$ represent the element $\alpha \beta$ of the transition density matrix between the Born-Oppenheimer states $\psi_{m}$ and $\psi_{n}$ in the molecular base $\{\alpha \beta\}, a_{p}{ }^{\alpha}$ are the SCF coefficients and $\varphi$ 's are the atomic orbitals. These couplings were solved by the central field method (CFM) ${ }^{16,29}$ where the dependence with the origin was eliminated.

\section{Results and Discussion}

The state-to-state charge transfer quantum cross sections were calculated by using a new method to solve the Schrödinger equation ${ }^{21}$ where the radial and rotational couplings can be taken into account. In this case, all couplings were included except the one with unphysical behavior.

The state-to-state charge transfer quantum cross sections and the LZ results are shown in table 1 for several collision energies. Because in the LZ calculation only two states $\left(2^{1} \Sigma\right.$ with $\left.3^{1} \Sigma\right)$ are taken into account, these cross sections are to be considered as the total cross section. For an analysis of these results, the quantum cross sections were split out for each allowed inelastic transition. The coupling between the $2^{1} \Sigma$ and $1^{1} \Pi$ states was neglected since in the electronic calculations this coupling generally does not have the correct asymptotic limit. This kind of procedure was also adopted recently by Boyd and co-workers ${ }^{15}$. Therefore, the coupling between these states are inferred from other couplings. As can be observed in Table 1, there is a weak coupling between the lowest $\left(1^{1} \Sigma\right)$ and highest $\left(3^{1} \Sigma\right)$ states used in our calculations and this is expected because such states have very weak radial coupling and no rotational coupling et al. The contribution due to the ionic and covalent channels, i.e., the $3^{1} \mathrm{Z}^{+}$state corresponding to the covalent and to the second ionic channel, were used in the LZ calculations. As can be seen in table 1 the LZ model describes correctly the general behaviour of the cross sec-

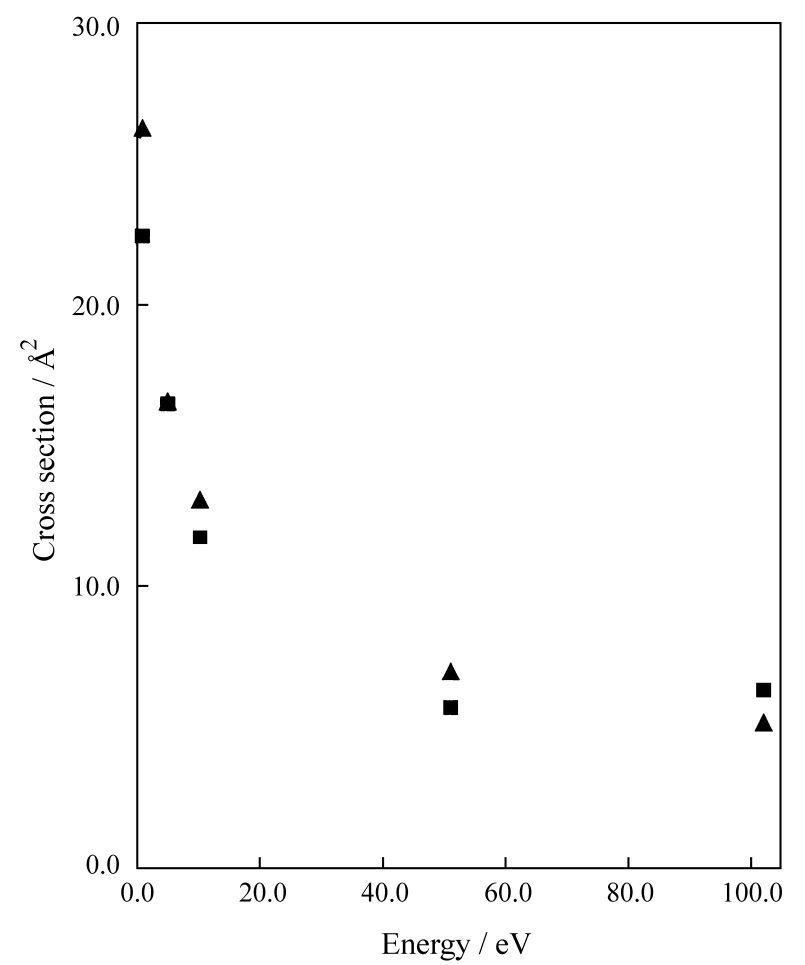

Figure 1. Comparison of total cross sections between our results (boxes) and Gardaud and co-workers ${ }^{22}$ cross sections (triangles).

Table 1. Cross sections in $\AA^{2}$. The LZ results are the total cross sections for the process under study. Subscripts 1,2 and 3 refer to the $\Sigma$ states and subscript 4 refers to the $\Pi$ state.

\begin{tabular}{lllll}
\hline$T / e V$ & $\sigma_{T}{ }^{L Z}$ & $\sigma_{13}{ }^{q}$ & $\sigma_{23}{ }^{q}$ & $\sigma_{34}{ }^{q}$ \\
\hline 1.0 & 83.70 & 0.0 & 2.576 & 1.145 \\
5.1 & 35.92 & 0.0 & 6.218 & 1.424 \\
10.3 & 25.18 & 0.001 & 5.942 & 1.646 \\
51.0 & 11.28 & 0.1786 & 3.158 & 1.858 \\
102.0 & 7.972 & 0.7063 & 3.372 & 1.915 \\
\hline
\end{tabular}


Table 2. Comparison of the potential parameters for some $a b$-initio calculations and the potential model where $\mathrm{R}_{\mathrm{c}}$ is the crossing point and $\Delta \mathrm{E}$ is the difference between the adiabatic potentials at the crossing.

\begin{tabular}{lcccc}
\hline & Inner $\mathrm{R}_{\mathrm{c}}$ & Crossing $\Delta \mathrm{E}$ & Outer $\mathrm{R}_{\mathrm{c}}$ & Crossing $\Delta \mathrm{E}$ \\
\hline Model potential(Gargaud et $_{\text {al. }}{ }^{22}$ ) & 4.6 & 0.095 & 7.4 & $3.0 \times 10^{-3}$ \\
Ab-initio (López-Castillo et al. $^{16}$ ) & 4.4 & 0.101 & 7.0 & $6.9 \times 10^{-3}$ \\
Ab-initio (Fraija et $_{\text {al. }}{ }^{26}$ ) & 4.4 & 0.100 & 7.0 & $5.5 \times 10^{-3}$ \\
Ab-initio (Shipsey et al. ${ }^{27}$ ) & 4.7 & 0.070 & 7.4 & $3.5 \times 10^{-3}$ \\
\hline
\end{tabular}

tion in this collision energy regime. For higher energies, corrections for this model have certainly to be taken into account. Moreover, it can also be observed from Table 1 that the charge transfer processes, for high energy collisions is not a two state problem. Figure 1 compares the total cross section out of the covalent state with the calculations of Gargaud and co-workers ${ }^{22}$. When the energy increases above $51 \mathrm{eV}$ the agreement becomes poorer and the main reason for this is certainly due to the absence of rotational coupling which is important for higher collision energies. Such a deviation can also be attributed to the difference

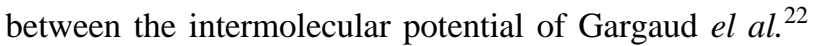
and the $a b$-initio potential of Lópes-Castillo and Ornellas ${ }^{16}$. This can be analysed by checking the potential parameters used in the two calculations as shown in Table 2 and compared with other calculations. The present results are equivalent to those of Fraija and co-workers and the greatest disagreement might be due to the small $\Delta \mathrm{E}$ of the outer crossing of the model potential ${ }^{22}$. In this case our $\Delta \mathrm{E}$ is almost twice that of the Gargaud results.

The important region for a charge transfer process is near the avoided crossing; although Gargaud et al. was more concerned with the difference of energy in the asymptotic region. It is recognized that the $\mathrm{CI}$ calculation is more reliable at intermediary $R$ than at the asymptotic limit. In the former case the potential model was parametrized for the asymptotic energies which have provided an error less than $0.0001 \%$. Therefore one can expect that the potential used in the present work can be more reliable for describing the charge transfer process than that reported by Gargaud and collaborators ${ }^{22}$, although they have good agreement for the collision energy range used in this work.

If the results of Fraija and co-workers ${ }^{30}$, and those reported by López-Castillo and Ornellas ${ }^{16}$ do not describe correctly the outer crossing, then the results of Shipsey et $a l .{ }^{31}$ also should not properly describe the system, since the three calculations employ the same method for the calculation. The agreement between Gargaud et al. calculations $^{22}$ with those of Shipsey and collaborators ${ }^{31}$, for the outer crossing, is likely to be incorrect. In addition, since the Shipsey et al. calculation is less accurate than those previouly reported ${ }^{16,30}$, it might be concluded that the results of Gargaud and co-workers are not quantitatively correct when compared with the calculations of Fraija et al. Moreover, Shipsey et al. have not used the translation factor and from their results ${ }^{16}$ (Fig. 6) it can be observed that the use of the central field method (or the use of the translation factor) will increase the absolute value of the total cross section ${ }^{16}$. Therefore, a comparison between the results of Gargaud and those of Shipsey will certainly disagree if such a factor is taken into account.

The comparison of the total quantum cross section against the results of Gargaud and co-workers ${ }^{22}$ in Fig. 1 shows that our results increase faster than those of Gargaud and co-workers. An extrapolation of these results for higher energies can show that the ab-initio cross section calculated in the present work will match the experimental results of Iwai and co-workers ${ }^{20}$. This can also be observed in the work of López-Castillo and Ornellas ${ }^{16}$ where they used a colinear classical path method. Actually, a simple extrapolation of the semi-classical total cross section presented by López-Castillo and Ornellas ${ }^{16}$ (Fig. 5) to lower energies indeed confirms this point.

\section{Acknowledgments}

We would like to thank the LNCC (Laboratório Nacional de Computação Científica) for providing the computational facilities where this calculation was partially carried out.

\section{References}

1. Bates, D.R.; McCarroll, R. Proc. R. Soc. London Ser. A 1958, 245, 175.

2.Delos, J.B.; Thorson, W.R. Phys. Rev. A 1978, 18, 117.

3. Gargaud, M.; Hanssen, J.; McCarroll, R; Valiron, P. J. Phys. B: At. Mol. Phys. 1981, 14, 2259.

4. Heil, T.G.; Butler, S.E.; Dalgarno, A. Phys. Rev. A 1981, 23, 1100.

5.Ng, C.Y.; Baer, M. Adv. Chem. Phys. 1992, 82.

6.Zewail, A.H. Science 1988 242, 1645.

7. Ng, C.Y. In Techniques for the Study of Ion-Molecule Reactions, Techniques of Chemistry, Farrar, J.N.; Saunders, W.H. Jr., Eds., New York 1988, vol. 20. 
8. Braga, J.P.; Knowles, D.B; Murrell, J. N. Molec. Phys. 1986, 57, 665.

9. Friedrich, B.; Pick, S.; Hládek, L.; Herman, Z.; Nikitin, E.E.; Reznikov, A.I.; Umanskii, S.Ya. J. Chem. Phys. 1986, 84, 807.

10. Herman, Z. Inter. Rev. Phys. Chem. 1996, 15, 299.

11. Herman, Z.; Jonathan, P.; Brenton, A.G.; Beynon, J.H. Chem. Phys. Lett. 1987, 141, 433.

12. Larsson, M.; Sigray, P. Chem. Phys. 1989, 139, 457.

13. Hamdan, M.; Brenton, A.G. J. Phys. B 1989, 22, L45.

14. Fárník, M.; Herman, Z.; Ruhaltinger, T.; Toennies, J.P. J. Chem. Phys. 1995, 103, 3495.

15. Boyd, R.; Ho, T.K.; Rabitz, H. J. Chem. Phys. 1997, 106, 6548.

16. López-Castillo, A.; Ornellas, F.R. Phys. Rev. A 1995, 51,381 .

17. López-Castillo, A. PhD Thesis, University of São Paulo, Institute of Chemistry 1992.

18. Zwally, H.J.; Cable, P.G. Phys. Rev. A 1971, 4, 2301.

19. Crandall, D.H. Phys. Rev. A 1977, 16, 958.

20. Iwai, T.; Kaneko, Y.; Kimura, M.; Kobayashi, N.; Ohtani, S.; Okuno, K.;Takagi, S.; Tawara, H.; Tsurubuchi, S. Phys. Rev. A 1982, 26, 105.

21. Braga, J.P.; Belchior, J.C. J. Comput. Chem. 1996, 17, 1559.
22. Gargaud, M.; Fraija, F.; Bacchus-Montabonel, M.C.; McCarroll, R. J. Phys. B: At. Mol. Phys. 1994, 7, 3985.

23. Fox, L.; Goodwin, E.T. Proc. Camb. Phil. Soc. 1949, 45, 373.

24. Abramowitz, M.; Stegun, I. Handbook of Mathematical Functions (Dover Publications, New York) 1972.

25. Barnett, A.R.; Feng, D.H.; Steed, J.W.; Goldfarb, L.J.B. Comput. Phys. Commun. 1974, 8, 377.

26. Dunning, T.H. Jr. J. Chem. Phys. 1971, 55, 716; 1989, 90, 1007.

27. Bacchus-Montabonel, M.C. Phys. Rev. A 1987, 36, 1994.

28. The MELD codes were developed by E.R.Davidson, L.E.Murchie, S.T.Elbert and S.R.Langhoff with extensive modifications by Rawlings, D.; Feller, D.; Davidson, E.R. in Modern Techniques in Computational Chemistry: MOTTEC-91, edited by Clementi, E. (Escom, Leiden, 1991), p. 381.

29. López-Castillo, A. Phys. Rev. A 1996, 53, 818.

30. Fraija, F.; Bacchus-Montabonel, M.C.; Gargaud, M. Z. Phys. D 1994, 29, 179.

31. Shipsey, E.J.; Browne, J.C.; Olson, R.E. Phys. Rev. A 1977, 15, 2166. 